\title{
Impacto da saúde mental materna na interação mãe-bebê e seus efeitos sobre o desenvolvimento infantil
}

\author{
Patrícia Alvarenga ${ }^{1}$ \\ Catiele Paixão ${ }^{1}$ \\ Zelma Freitas Soares ${ }^{1}$ \\ Antonio Carlos Santos da Silva ${ }^{1}$ \\ ${ }^{1}$ Universidade Federal do Bahia, BA, Brasil
}

\begin{abstract}
Resumo
Os prejuízos na saúde mental da mãe podem afetar seus comportamentos em interações com o bebê, que são importantes preditores do desenvolvimento infantil no primeiro ano de vida. Este estudo investigou o impacto da depressão pós-parto e da ansiedade na interação mãe-bebê e seus efeitos no desenvolvimento aos três meses de vida. Participaram do estudo 64 díades mãe-bebê que foram filmadas em um episódio de brincadeira livre. As mães responderam ao SRQ-20 e ao BDI para avaliação da saúde mental. O desenvolvimento dos bebês foi avaliado com a EDCC. As análises de regressão confirmaram o poder preditivo da depressão sobre a frequência de sorrisos maternos e também da estimulação tátil ou com objetos sobre os comportamentos motores do bebê. As evidências do impacto da depressão pós-parto sobre os comportamentos sensíveis da mãe e da estimulação não-verbal sobre o desenvolvimento motor nos primeiros meses de vida são discutidas.
\end{abstract}

Palavras-chave: Depressão pós-parto; Ansiedade; Relações mãe-criança; Desenvolvimento infantil; Desenvolvimento motor.

\section{Impact of maternal mental health in mother-infant interaction and its efects on child development}

\begin{abstract}
Maternal mental health impairments can affect mothers' behavior during interactions with the baby, which are important predictors of childhood development in the first year. This study investigated the impact of postpartum depression and anxiety on motherinfant interaction and its developmental effects at 3 months of age. Participants were 64 mother-baby dyads that were filmed in a free-play episode. Mothers responded to SRQ-20 and BDI for mental health assessment. Babies' development was evaluated with EDCC. Regression analyzes confirmed the predictive power of depression on the frequency of maternal smiles and also of tactile or object stimulation on baby's motor behavior. The evidence of the impact of postpartum depression and nonverbal stimulation during interaction on the mother's sensitive behaviors and motor development in the first months of life are discussed.
\end{abstract}

Keywords: Postpartum depression; Anxiety; Mother-child relations; Childhood development; Motor development.

\section{Impacto de la salud mental materna en la interacción madre-bebé y sus efectos sobre el desarrollo infantil}

\section{Resumen}

Daños en la salud mental de la madre pueden afectar sus comportamientos en las interacciones con el bebé, que son predictores del desarrollo infantil en el primer año de vida. Este estudio investigó el impacto de la depresión postparto y de la ansiedad en la interacción madre-bebé y sus efectos en el desarrollo a los tres meses de vida. Participaron 64 díadas madre-bebé, filmadas en un episodio juego libre. Las madres respondieron al SRQ-20 y al BDI para la evaluación de la salud mental. El desarrollo de los bebés fue evaluado con la EDCC. Análisis de regresión confirmaron el poder predictivo de la depresión sobre la frecuencia de sonrisas maternas y también de la estimulación táctil o con objetos sobre los comportamientos motores del bebé. Evidencias del impacto de la depresión posparto y de la estimulación no verbal durante la interacción sobre comportamientos sensibles de la madre y el desarrollo motor en los primeros meses de vida son discutidas.

Palabras clave: Depresión post-parto; Ansiedad; Relaciones madre-niño; Desarrollo infantil; Desarollo motor. 


\section{Introdução}

A qualidade da interação mãe-bebê é essencial para o desenvolvimento linguístico (Bornstein, Putnick, Cote, Haynes, \& Suwalsky, 2015), socioemocional (Karam et al., 2016), cognitivo (Pereira, Valentini, \& Saccani, 2016) e motor (Saccani, Valentini, Pereira, Müller, \& Gabbard, 2013) nos primeiros meses de vida. A interação mãe-bebê é definida como um tipo de contexto no qual mãe e bebê se relacionam exibindo respostas comportamentais um ao outro, de forma mútua, e tem sido comumente avaliada através dos padrões de sensibilidade ou responsividade da mãe aos sinais do bebê (Brummelte \& Galea, 2016). Os comportamentos maternos sensíveis ou responsivos podem ser definidos com base nas respostas contingentes, apropriadas e não intrusivas da mãe aos sinais do bebê, que favorecem a exploração do ambiente, a formação de vínculos afetivos e as relações interpessoais em geral (Isabella, Belsky, \& Von Eye, 1989). De modo contrário, mães que interagem de forma menos sensível, intrusiva ou apática aos sinais e demandas do lactente tendem a dificultar comportamentos exploratórios e a formação de vínculos por parte da criança (Choi, 2013; Edwards \& Hans, 2016; Parfitt, Pike, \& Ayers, 2013), especialmente por não garantirem a segurança, o tempo e o espaço necessários para as expressões comportamentais do bebê ou por não fornecerem estímulos importantes para a aquisição de novas habilidades (Alvarenga, Weber, \& Bolsoni-Silva, 2016). Nesta perspectiva, a Teoria do Apego pressupõe que o tipo de vinculação afetiva estabelecida entre a mãe e a criança exerce influência relevante sobre o desenvolvimento infantil. Crianças que experimentam cuidados pouco responsivos tendem a desenvolver um padrão de apego inseguro, que está relacionado à falta de confiança na disponibilidade emocional dos cuidadores e, consequentemente, ao menor nível de confiança em relação às interações sociais e ao ambiente em geral. Por outro lado, cuidados sensíveis e responsivos tendem a favorecer um padrão de apego seguro que, por sua vez, repercute em uma orientação positiva e confiante por parte da criança quanto às relações interpessoais e ao mundo ao seu redor (Bowlby, 1969).

Durante o primeiro ano de vida, o desenvolvimento da criança ocorre em ritmo acelerado e diversas mudanças no comportamento do bebê podem ser observadas ao longo de cada mês (Pinto, Vilanova, \& Vieira, 1997). Aos três meses, as mudanças mais expressivas são observadas no desenvolvimento motor, quando é esperado que o bebê seja capaz de tentar levar a mão à boca, segurar um objeto por ao menos dois segundos, girar levemente a cabeça para observar algo que lhe chama atenção e sustentar a cabeça quando colocado em posição ventral. Alguns aspectos do desenvolvimento cognitivo também já podem ser percebidos, por exemplo, quando o bebê é capaz de reconhecer o cuidador, observar algo por alguns segundos e demonstrar preferência por objetos. No que se refere à linguagem, aos três meses o bebê já começa a balbuciar emitindo sons agudos ou pronunciando sons de vogais como "ahhh" e é capaz de se acalmar quando o cuidador fala com ele. Além dessas mudanças, como característica do desenvolvimento socioemocional, é esperado que o bebê sorria e reaja às expressões emocionais dos outros, sinalizando seus estados de humor (Bayley, 2006).

Além de orientar o ritmo da interação a partir das pistas comportamentais que o bebê fornece nesses contextos, as mães também precisam ser sensíveis e atentas às capacidades e necessidades do bebê a cada fase do desenvolvimento para, assim, estimulá-lo adequadamente. Por exemplo, à medida que o lactente cresce, torna-se importante aumentar os desafios para sua adaptação postural e mães que posicionam os bebês de forma a garantir maior independência e liberdade dos movimentos, ao invés de mantê-los apenas em posições com apoio, tendem a favorecer seu desenvolvimento motor (Pereira et al., 2016; Saccani et al., 2013). De forma semelhante, mães que utilizam brinquedos com maiores níveis de complexidade durante as interações, de acordo com crescimento do lactente, e que garantem maior diversidade de espaço físico para a interação com o bebê favorecem seu desenvolvimento cognitivo (Mermelshtine \& Barnes, 2016; Pereira et al., 2016). Um estudo realizado no Brasil e em outros países mostrou que quando as mães interagem falando com seus bebês com maior frequência eles também respondem com vocalizações mais frequentes (Bornstein et al., 2015). Esse estudo mostra o impacto da estimulação e das reações maternas aos comportamentos dos bebês sobre seu desenvolvimento, desde o nascimento até os 18 meses de vida.

Vários fatores afetam os padrões de interação que se estabelecem entre mães e filhos no primeiro ano de vida. Existem evidências consistentes a respeito do impacto de variáveis sociodemográficas na interação mãe-bebê e no desenvolvimento da criança. Bebês de famílias socioeconomicamente vulneráveis, aquelas que possuem menor poder aquisitivo e menores níveis de escolaridade, tendem a apresentar atrasos no desenvolvimento cognitivo que podem ser constatados desde os primeiros meses de vida (Clearfield, Stanger, \& Jenne, 2015; Koutra et al., 2013; Mermelshtine \& Barnes, 2016). De modo mais específico, a escolaridade dos pais, a coabitação parental e a renda familiar têm 
sido relacionadas ao desenvolvimento cognitivo e motor. Fatores como maiores renda e escolaridade, além de espaço físico mais amplo para as interações, estão associados ao maior repertório de habilidades linguísticas e sociais das mães para interagirem com o bebê (Mermelshtine \& Barnes, 2016; Pereira et al., 2016; Saccani et al., 2013).

A saúde mental materna é outro preditor significativo dos padrões de interação mãe-bebê e do desenvolvimento da criança. Diferentes construtos e instrumentos têm sido empregados na investigação dos problemas da saúde mental materna no período pós-parto e ao longo do primeiro ano do bebê. Entre eles destacam-se os conceitos de depressão pós-parto e de transtornos mentais comuns (Alvarenga, Dazzani, Lordelo, Alfaya, \& Piccinini, 2013; Kingston, Tough, \& Whitfield, 2012). A depressão pós-parto corresponde a um quadro de depressão maior iniciado durante as primeiras quatro semanas após o nascimento do bebê (APA, 2014). Quanto aos transtornos mentais comuns, este conceito inclui sintomas de ansiedade, depressão e queixas somáticas que não atendem aos critérios formais para o diagnóstico de ansiedade ou depressão, conforme classificações nosológicas, mas que representam comprometimento do funcionamento psicológico do indivíduo, semelhante ou até mesmo pior do que os quadros crônicos já bem estabelecidos (Santos, 2007).

Uma revisão sistemática da literatura indicou alta prevalência de depressão pós-parto entre brasileiras e mostrou que mães de populações carentes são mais frequentemente afetadas, com prevalência entre 30 e $40 \%$, índice bastante superior àquele encontrado entre mães com maior nível socioeconômico, que é de cerca de 20\% (Lobato, Moraes, \& Reichenheim, 2011). Esses dados são relevantes para profissionais de saúde por conta das repercussões da depressão pós-parto nos comportamentos da mãe durante as interações com o filho. Alguns estudos destacam que mães com depressão são menos propensas a apresentar comportamentos maternos sensíveis aos sinais e necessidades de seus bebês (Brummelte \& Galea, 2016; Choi, 2013; Edwards \& Hans, 2016). Por exemplo, comparadas às mães sem prejuízos emocionais, mães com depressão pós-parto tendem a se engajar menos nas interações com o lactente e a apresentar menor quantidade de estímulos afetuosos direcionados ao bebê como sorrir, interagir face-a-face, tocar, narrar histórias e falar (Brummelte \& Galea, 2016). Além da escassez de comportamentos afetuosos, mães com depressão pós-parto também tendem a apresentar menor sensibilidade ao ritmo comportamental mais lento do bebê e assim, frequentemente, agem de forma intrusiva, o que, por sua vez, pode repercutir em uma menor disposição do lactente para interações sociais, além de limitar suas oportunidades para treinar novas habilidades (Weikum, Mayes, Grunau, Brain, \& Oberlander, 2013). A intrusividade pode ser observada nos comportamentos maternos que interrompem os comportamentos do bebê, limitando seus movimentos e demonstrando pouca sensibilidade à sua necessidade crescente de explorar objetos no ambiente.

Os prejuízos na interação mãe-bebê tendem a variar em função do grau de comprometimento da saúde mental materna (Kingston et al., 2012), porém, mesmo em populações com sintomas de depressão menos graves, a interação pode ser prejudicada. Apesar de não apresentarem diferenças na frequência de expressões faciais positivas ou negativas durante uma interação face-a-face com bebês de três meses de vida, mães com baixos escores de depressão foram menos propensas a adaptar seus comportamentos de forma diversificada para interações contingentes e não-contingentes com seus filhos, ou seja, as mães apresentavam as mesmas expressões faciais de afeto positivo e negativo, independentemente das expressões apresentadas pelos bebês (Braarud et al., 2017). O impacto da depressão pós-parto pode ser ainda mais grave em mães com outros prejuízos emocionais como o estresse e a ansiedade. Mulheres com sintomas de ansiedade durante o pré-natal tendem a ser mais controladoras e menos sensíveis aos sinais de seus bebês nos primeiros meses de vida e, nesse contexto, os lactentes tendem a responder de forma mais passiva do que ativa durante as interações (Parfitt et al., 2013). $\mathrm{O}$ estresse materno também pode acarretar atrasos no desenvolvimento cognitivo e psicomotor (Kingston et al., 2012).

Os achados de uma revisão sistemática sobre o impacto do sofrimento psicológico materno, incluindo ansiedade, estresse e depressão, no desenvolvimento de bebês de até 12 meses de vida indicaram diferentes repercussões no desenvolvimento infantil em função do período de sofrimento psicológico. Os prejuízos na saúde mental durante o pré-natal estiveram associados a problemas ou atrasos no desenvolvimento psicomotor, cognitivo e comportamental e o sofrimento psicológico durante o pós-natal foi associado a atrasos ou problemas no desenvolvimento cognitivo e socioemocional da criança (Kingston et al., 2012). Outros estudos publicados posteriormente apoiam a hipótese de que diferentes problemas de saúde mental materna estão associados a prejuízos no desenvolvimento motor, cognitivo e socioemocional da criança (Karam et al., 2016; Koutra et al., 2013). 
A literatura revisada mostra a relevância de estudos que investiguem as relações entre a saúde mental materna e o desenvolvimento do bebê, especialmente porque, como as evoluções nos comportamentos do lactente acontecem em ritmo acelerado, as interações mãebebê que ocorrem desde os primeiros meses podem ter impacto nas diversas dimensões do desenvolvimento. Ademais, os achados de investigações sobre essa problemática em populações socioeconomicamente vulneráveis apontam para a importância de estudos dessa natureza no Brasil. Desse modo, a proposta do presente estudo foi investigar, em uma amostra de díades socialmente vulneráveis, o impacto da saúde mental da mãe em seus comportamentos durante a interação com o bebê e avaliar as relações entre os comportamentos maternos e os indicadores do desenvolvimento aos três meses de vida. Desse modo, o objetivo do presente estudo foi investigar, em uma amostra de díades socialmente vulneráveis, o impacto da saúde mental da mãe em seus comportamentos durante a interação com o bebê e avaliar as relações entre os comportamentos maternos e os indicadores de desenvolvimento aos três meses de vida. A primeira hipótese testada foi a de que maiores escores de depressão pós-parto e de transtornos mentais comuns seriam preditores de menor frequência de comportamentos maternos sensíveis e de maior frequência de comportamentos intrusivos da mãe. A segunda hipótese era de que a maior frequência de comportamentos maternos sensíveis e a menor frequência de comportamentos maternos intrusivos seriam preditores de maiores escores dos indicadores de desenvolvimento do bebê.

\section{Método}

\section{Participantes}

Participaram do estudo 64 díades mãe-bebê, recrutadas junto a Unidades de Saúde da Família de duas comunidades socioeconomicamente vulneráveis na cidade de Salvador, Brasil. Essa amostra foi selecionada para um estudo longitudinal mais amplo que avaliou os efeitos de uma intervenção para aumentar o nível de responsividade materna e favorecer o desenvolvimento da criança. Os bebês participantes do presente estudo (39 do sexo masculino e 25 do sexo feminino) tinham três meses de vida, nasceram a termo e não apresentavam enfermidades crônicas físicas ou mentais. As mães tinham, aproximadamente, 26 anos de idade $(\mathrm{M}=26,53 ; \mathrm{DP}=5,55)$, estudaram, em média, 10,05 anos $(\mathrm{M}=10,05 ; \mathrm{DP}=2,89)$, que corresponde ao ensino médio incompleto, e 57,8\% delas não trabalhavam fora de casa. Os pais dos bebês também estudaram, aproximadamente, 10 anos $(\mathrm{M}=10,53 ; \mathrm{DP}=2,47)$ e $79,7 \%$ deles exerciam alguma atividade remunerada fora de casa. A maior parte das mães vivia com o pai do bebê $(78,1 \%)$ e não apresentava problemas de saúde física $(87,5 \%)$. Apenas oito mães relataram problemas de saúde física e mencionaram sintomas relacionados a problemas de tireoide, anemia, hipertensão, lúpus reumática, epilepsia, rinite ou anemia falciforme. A renda familiar foi de aproximadamente dois salários mínimos em reais $(\mathrm{M}=1408,91 ; D P=830,47)$, conforme vigência no período da coleta de dados. Mais detalhes sobre as características sociodemográficas podem ser consultadas na Tabela 1 .

TABELA 1

Características Sociodemográficas das Participantes $(N=64)$

\begin{tabular}{|c|c|c|c|c|c|}
\hline \multirow{2}{*}{ Variáveis } & \multirow{2}{*}{ Categorias } & \multicolumn{4}{|c|}{ Valores } \\
\hline & & $n$ & $\%$ & $M$ & $D P$ \\
\hline \multirow[t]{2}{*}{ Sexo do bebê } & Masculino & 39 & 60,9 & & \\
\hline & Feminino & 25 & 39,1 & & \\
\hline Idade da mãe do bebê (em anos) & & & & 26,53 & 5,55 \\
\hline Escolaridade da mãe do bebê (em anos) & & & & 10,05 & 2,89 \\
\hline \multirow[t]{2}{*}{ Ocupação da mãe do bebê } & Trabalham fora & 27 & 42,2 & & \\
\hline & Não trabalham fora & 37 & 57,8 & & \\
\hline \multirow[t]{2}{*}{ Problemas de saúde da mãe } & Sim & 8 & 12,5 & & \\
\hline & Não & 56 & 87,5 & & \\
\hline Quantidade de filhos & & & & 0,69 & 0,89 \\
\hline Escolaridade do pai do bebê (em anos) & & & & 10,53 & 2,47 \\
\hline \multirow[t]{2}{*}{ Ocupação do pai do bebê } & Trabalham fora & 51 & 79,7 & & \\
\hline & Não trabalham fora & 11 & 17,2 & & \\
\hline \multirow[t]{2}{*}{ Coabitação parental } & Sim & 50 & 78,1 & & \\
\hline & Não & 14 & 21,9 & & \\
\hline Tempo da união conjugal (em meses) & & & & 44,67 & 44,63 \\
\hline Renda familiar (em reais) & & & & 1408,91 & 830,47 \\
\hline
\end{tabular}




\section{Instrumentos}

Ficha de Dados Sociodemográficos: Foi confeccionada especialmente para este estudo, com o objetivo de investigar dados como sexo do bebê, idade da mãe, escolaridade, profissão, renda familiar e existência de outros filhos.

Self-Report Questionnaire of Minor Psychiatric Disorders (SRQ-20): Desenvolvido por Harding et al. (1980, citado por Mari \& Williams, 1986), trata-se de uma escala de rastreamento psiquiátrico composta por 20 itens que avaliam sintomas não psicóticos de transtornos psiquiátricos menores ou comuns, a partir de respostas 'sim' ou 'não'. Investigou-se, portanto, a presença de sintomas de depressão, ansiedade e queixas psicossomáticas. Para este estudo foi usada a versão brasileira da escala, cujo índice de consistência interna (alpha de Cronbach) foi igual a 0,86 (Gonçalves, Stein, \& Kapczinski, 2008). A soma dos itens com resposta "sim" corresponde ao escore total na escala, que pode variar de zero a 20 pontos. Quanto maior o escore, maior a probabilidade de presença de algum transtorno mental comum. Escores acima de oito pontos indicam risco de transtorno mental.

Inventário Beck de Depressão I (BDI-I): Este instrumento é uma escala sintomática de autorrelato, desenvolvida por Beck e Steer, (1993). É composta por 21 itens com diferentes alternativas de resposta sobre como o indivíduo tem se sentido recentemente, e que correspondem a níveis diferentes de gravidade dos sintomas de depressão. Os itens incluem afirmativas como, por exemplo, "não encontro um prazer real em mais nada" ou "eu me sinto sempre culpado". A soma dos escores dos itens individuais fornece um escore total, que pode variar de zero a 63 pontos e constitui um escore dimensional da intensidade dos sintomas de depressão. Esses escores podem ser classificados em quatro níveis: mínimo, para escores de até 11 pontos; leve, para escores entre 12 e 19 pontos; moderado, para escores entre 20 e 35 pontos; ou grave, para escores a partir de 36 pontos. Assim, quanto maior o escore, mais relevantes os sintomas de depressão. Para o presente estudo, foi utilizada a versão em português do Inventário (Cunha, 2001), cuja consistência interna (alpha de Cronbach) foi 0,84 .

Observação da Interação Mãe-Bebê - 30 mês: A díade mãe-bebê foi filmada em um episódio de interação utilizando três brinquedos fornecidos pelos pesquisadores: um chocalho, um boneco de borracha e uma pequena bola de borracha. Foram analisados os seis minutos iniciais de cada vídeo, em intervalos de 12 segundos, com base em categorias descritas na literatura (Bornstein, Tamis-LeMonda, Chun-Shin,
\& Haynes, 2008; Piccinini, Alvarenga, \& Frizzo, 2007). Nos primeiros seis segundos foram registradas as ocorrências dos comportamentos do bebê em três categorias distintas: a) sorri (o bebê visivelmente sorri, sendo incluídos também sorrisos não dirigidos à mãe); b) emite sons (o bebê balbucia ou mexe a boca, emitindo sons vocálicos ou não vocálicos, fazendo tentativas de vocalização ou imitação da vocalização materna, sendo incluídas também vocalizações de protesto e "risadas"); e c) chora/choraminga (o bebê encontra-se visivelmente desconfortável, inquieto e/ou chora, sendo incluído também o choro contínuo e com forte intensidade).

As ocorrências das respostas maternas aos comportamentos do bebê foram registradas no mesmo intervalo e no intervalo seguinte (de seis segundos) em seis categorias distintas: a) interpreta/fala pelo bebê (a mãe vocaliza, colocando-se empaticamente no lugar do bebê, interpretando o estado e/ou os sinais do bebê); b) fala para o bebê (refere-se exclusivamente a verbalizações da mãe, cujo conteúdo não envolve a brincadeira. Nessa categoria, a mãe vocaliza, falando com o bebê, imitando suas vocalizações, cantando para ele ou emitindo sons); c) sorri para o bebê (a mãe sorri, dirigindo seu olhar para o bebê e ambos estão no mesmo plano visual); d) pega no colo/embala/ aconchega (a mãe pega o bebê no colo e/ou balança o bebê em seus braços ou pernas; traz o bebê junto de seu corpo); e) toca/estimula fisicamente/estimula com objeto (a mãe toca o bebê com partes de seu corpo para estimulá-lo ou faz gestos com ou sem o auxílio de objetos/brinquedos para chamar a atenção do bebê); e, f) age de forma intrusiva (comportamentos maternos que interrompem os comportamentos do bebê, que limitam seus movimentos e acesso aos objetos do ambiente, ou ainda que provocam estimulação tátil, auditiva ou visual excessiva).

As categorias (a), (b), (c), (d), e (e) incluem comportamentos maternos considerados sensíveis, enquanto a categoria (f) foi utilizada para o registro de todas as respostas maternas consideradas intrusivas. Foram registrados todos os comportamentos das mães e dos bebês, no entanto, para esse estudo foram analisados apenas os comportamentos da mãe. Os comportamentos maternos registrados eram mutuamente excludentes, ou seja, um mesmo comportamento não foi registrado em mais de uma categoria. Após o registro da ocorrência dos comportamentos maternos em cada um dos intervalos de seis segundos, foram calculadas as frequências dos comportamentos maternos em cada uma das categorias de análise. A análise da interação mãe-criança foi realizada por dois codificadores independentes, que receberam treinamento prévio. A fidedignidade foi 
estabelecida com base em 10 vídeos e atingiu 0,77 para as categorias de comportamentos maternos e 0,68 para as categorias de comportamentos infantis (coeficiente Kappa de Cohen). Esses valores são considerados, respectivamente, excelente e bom (Robson, 1993). Para as análises estatísticas realizadas foram utilizadas apenas as frequências de comportamentos maternos durante a interação.

Escala de Desenvolvimento do Comportamento da Criança (EDCC): Desenvolvida no Brasil por Pinto et al. (1997), trata-se de um instrumento que, através de uma interação lúdica com a criança, permite a avaliação do desenvolvimento do comportamento motor e do comportamento atividade de crianças de um a 11 meses de idade. A escala considera os comportamentos de cada mês nesta faixa etária e fornece uma indicação do ritmo de desenvolvimento e uma avaliação qualitativa do processo de desenvolvimento do comportamento da criança. A EDCC analisa 64 comportamentos observados no primeiro ano de vida e faz distinção entre comportamentos motores e comportamento atividade. Os comportamentos motores, que envolvem respostas como levar a mão à boca ou engatinhar, são subdivididos quanto ao eixo somático e à estimulação. No que se refere ao eixo somático, os comportamentos motores pertencem à categoria axial, que corresponde ao eixo longitudinal crânio-caudal, ou à categoria apendicular, que contempla movimentos com os membros inferiores e superiores. Quanto à estimulação, esses comportamentos são subdivididos em estimulado ou espontâneo. Quanto aos comportamentos atividade, aqueles que se referem às respostas em busca de interação social, como emitir sons vocálicos ou virarse quando chamado pelo nome, são subdivididos em comportamentos comunicativos ou não comunicativos. Nesse instrumento, os comportamentos também estão agrupados em função da probabilidade de ocorrência, a cada mês de idade e para cada sexo, de modo que são classificados em três categorias: a) aparecimento, agrupa comportamentos que não são típicos daquela fase do desenvolvimento, mas que podem estar presentes, pois foram observados em pelo menos uma criança daquela faixa etária; b) normalização, reúne comportamentos apresentados por cerca de 50\% das crianças naquela faixa etária; e, c) estabilização, agrupa comportamentos presentes em aproximadamente $90 \%$ das crianças daquela faixa etária. Com essa classificação é possível observar as idades, em meses, nas quais cada comportamento tende a aparecer (aparecimento), a ser comum (normalizar) e a ser altamente frequente (estabilizar-se), permitindo uma indicação do ritmo de desenvolvimento e possibilitando uma estimativa acerca de atrasos ou aceleração do desenvolvimento.
A soma dos comportamentos apresentados em cada uma das três categorias foi considerada como o escore na categoria em questão. A partir desse escore foram geradas proporções nas categorias aparecimento, normalização e estabilização, dividindo-se o total de comportamentos observados em cada categoria pelo número total de itens da escala na referida categoria para os meninos e para as meninas. Essas proporções foram utilizadas como escores ponderados das três categorias em todas as análises realizadas.

\section{Procedimentos}

Coleta de dados. Após aprovação pelo Comitê de Ética em Pesquisa da Faculdade de Medicina da Bahia, da Universidade Federal da Bahia (Parecer no 396.333), famílias de gestantes cadastradas em Unidades de Saúde da Família de duas comunidades socioeconomicamente vulneráveis, em Salvador-Brasil, foram convidadas para participar do estudo longitudinal. Os dados do presente estudo correspondem a uma parte da avaliação que foi realizada antes de iniciar a intervenção do estudo longitudinal anteriormente mencionado. As mães que concordaram com a participação receberam uma visita domiciliar, quando o bebê tinha três meses de vida, na qual forneceram informações sociodemográficas da família e responderam ao SRQ-20 e ao BDI em forma de entrevista, ou seja, o pesquisador lia os itens e as alternativas de resposta e a mãe informava a resposta escolhida, com auxílio de um cartão de respostas. $\mathrm{Na}$ mesma visita também foi realizada a Observação da Interação Mãe-Bebê e, em seguida, foi realizada a aplicação da EDCC. Os pesquisadores responsáveis por esta avaliação foram treinados para a aplicação dos instrumentos e realização das filmagens. Além disso, realizaram testes piloto antes de iniciarem a coleta de dados.

Análise dos dados. Inicialmente foram realizadas análises descritivas, incluindo mínimo, máximo, média, desvio padrão e intervalo de confiança (95\%), dos indicadores do desenvolvimento do bebê e das variáveis preditoras investigadas. Em seguida, foram utilizados testes de correlação bivariada e de regressão linear. Os testes de Pearson e Spearman foram adotados de acordo com a normalidade da distribuição dos dados de cada, que foi verificada nos histogramas de cada uma das variáveis consideradas. Apenas as variáveis BDI, comportamentos infantis em aparecimento e três categorias de comportamentos maternos, pega no colo/embala/aconchega, toca ou estimula com objetos e age de forma intrusiva, apresentaram curva simétrica. A partir dos resultados das análises de correlação, modelos de regressão linear stepwise foram testados com as variáveis que estiveram correlacionadas para confirmar seu poder preditivo. 


\section{Resultados}

As análises de estatística descritiva indicaram que os escores no BDI variaram entre 1 e 51 , com média de $15,81(\mathrm{DP}=10,42 ; \mathrm{IC}=13,19-18,33)$. De acordo com os pontos de corte do instrumento, os relatos de $35,9 \%$ das mães sugeriram relevância clínica para depressão. O escore médio do SRQ-20 foi igual a 4,78 $(\mathrm{DP}=3,79$; $\mathrm{IC}=3,84-5,72)$ e os escores nessa escala variaram entre 0 e 15 . Com base no ponto de corte do instrumento, os relatos de $25 \%$ das mães apontaram para a possível presença de transtornos mentais. Para investigar a frequência dos comportamentos da mãe, foram efetuadas análises descritivas da ocorrência de cada uma das categorias de comportamentos maternos. A categoria mais frequente foi fala para o bebê, com média de ocorrência igual a 44,37 $(\mathrm{DP}=14,15 ; \mathrm{IC}=40,59-47,66)$, seguida pelas categorias pega no colo/embala/aconchega $(\mathrm{M}=30,78$; $\mathrm{DP}=27,20 ; \mathrm{IC}=23,84-37,37)$, toca ou estimula com objetos $(\mathrm{M}=30,46 ; \mathrm{DP}=8,40 ; \quad \mathrm{IC}=28,45-32,51)$ e age de forma intrusiva $(\mathrm{M}=28,66 ; \mathrm{DP}=10,17$; $\mathrm{IC}=26,24-31,25)$. A proporção das categorias sorri para o bebê $(\mathrm{M}=8,10 ; \mathrm{DP}=10,82 ; \mathrm{IC}=5,57-10,69)$ e interpreta/fala pelo bebê $(\mathrm{M}=3,96 ; \mathrm{DP}=4,78$; $\mathrm{IC}=2,85-5,23$ ) foram menos frequentes. Quanto aos indicadores do desenvolvimento infantil, a proporção de comportamentos em estabilização variou entre 40 e $100 \%$ e o percentual médio de ocorrências desses comportamentos foi igual a 90,31\% (DP=13,33\%; $\mathrm{IC}=86,88-93,44 \%)$. A proporção de comportamentos em normalização variou entre 0 e $100 \%$ e o percentual médio de ocorrências desses comportamentos foi de $85,63 \%(\mathrm{DP}=21,30 \%$; IC =80,16-90,63\%). Por fim, a proporção de comportamentos em aparecimento variou entre 25 e $83 \%$, com média igual a 55,94\% $(\mathrm{DP}=13,42 \%$; IC $=52,92-59,40 \%)$.

\section{Saúde mental materna e comportamentos maternos durante a interação mãe-bebê}

Os resultados revelaram correlações entre a saúde mental da mãe e os comportamentos por ela apresentados durante as interações com o bebê (Tabela 2). O teste de Spearman evidenciou correlações negativas entre os sintomas de transtornos mentais comuns e os comportamentos de sorrir para o bebê $(\rho=-0,23$; $p<0,05)$ e de tocar o bebê ou estimulá-lo com objetos $(\rho=-0,25 ; p<0,05)$. Assim, quanto mais sintomas de transtornos mentais comuns relatados pelas mães, menos frequentes foram as ocasiões em que elas sorriam para o bebê e o tocavam ou estimulavam com objetos. O teste de Spearman também indicou uma correlação negativa entre os sintomas de depressão e os comportamentos de sorrir para o bebê $(\rho=-0,36$; $p<0,01)$, ou seja, quanto mais sintomas de depressão, menos as mães sorriam para o bebê. Por fim, o teste de Pearson apontou para uma correlação negativa entre os sintomas de depressão e a prática de tocar ou estimular o bebê com objetos $(\mathrm{r}=-0,22 ; p<0,05)$, indicando que quanto mais sintomas de depressão relatados pela mãe, menos frequentes foram os comportamentos de tocar ou estimular o bebê com objetos. As demais correlações entre a saúde mental e os comportamentos maternos durante a interação da díade mãe-bebê não foram significativas.

TABELA 2

Correlações entre Transtornos Mentais Comuns (SRQ-20), Sintomas de Depressão (BDI) e os comportamentos da mãe durante a interação com o bebê $(N=64)$

\begin{tabular}{lcc}
\hline Comportamentos da mãe & $S R Q-20$ & $B D I$ \\
Interpreta/fala pelo bebê & 0,11 & $-0,11$ \\
Fala para o bebê & 0,03 & $-0,07$ \\
Sorri para o bebê & $-0,23^{*}$ & $-0,36^{* *}$ \\
Pega no colo/embala/aconchega & 0,12 & 0,16 \\
Toca ou estimula com objetos & $-0,25^{*}$ & $-0,19^{*}$ \\
Age de forma intrusiva & 0,11 & 0,13 \\
\hline
\end{tabular}

$* p<0,05 ; * * p 0,01$.

Um primeiro modelo de regressão testou o poder preditivo dos transtornos mentais comuns e da depressão pós-parto sobre a frequência de sorrisos da mãe. A análise de regressão (stepwise) confirmou o poder preditivo da depressão pós-parto, que explicou 10,9\% da variância $\left(\mathrm{R}^{2}=0,109\right)$ na frequência de sorrisos maternos $(F(2)=4,87 ; \mathrm{p}=0,01)$. A cada sintoma a mais no BDI (depressão pós-parto), o número de sorrisos maternos durante a interação diminuiu em 0,42 ocorrências. Os transtornos mentais comuns foram excluídos do modelo $(\mathrm{p}=0,68)$. Esses resultados confirmam o poder preditivo da depressão pós-parto sobre os sorrisos da mãe durante a interação, porém, não confirmam o impacto dos transtornos mentais comuns sobre essa variável. O segundo modelo testado avaliou o poder preditivo dos transtornos mentais comuns e da depressão pós-parto sobre a frequência de estimulação tátil e com objetos, porém, os resultados da análise não foram significativos $(F(1)=3,14 ; \mathrm{p}=0,81)$. Esses achados não confirmam o impacto dessas variáveis na variância da estimulação tátil e com objetos durante a interação mãe-bebê.

\section{O impacto dos comportamentos maternos sobre o desenvolvimento do bebê}

Os achados da avaliação das correlações entre os comportamentos maternos durante a interação com o bebê e os comportamentos do bebê em 
TABELA 3

Correlações entre os Comportamentos da Mãe Durante a Interação com o Bebê e os Comportamentos Infantis em Estabilização, Normalização e Aparecimento $(N=64)$

\begin{tabular}{lccc}
\hline Comportamento Materno & Estabilização & Normalização & Aparecimento \\
Interpreta/fala pelo bebê & $-0,06$ & 0,18 & 0,15 \\
Fala para o bebê & 0,03 & 0,16 & 0,03 \\
Sorri para o bebê & $-0,10$ & 0,07 & 0,06 \\
Pega no colo/embala/aconchega & 0,12 & $-0,08$ & 0,06 \\
Toca ou estimula com objetos & $0,26 *$ & 0,11 & $-0,03$ \\
Age de forma intrusiva & $-0,22 *$ & 0,00 & 0,00 \\
\hline$* 0,05 ; * *<0,01$. & &
\end{tabular}

estabilização, normalização ou aparecimento podem ser observados na Tabela 3. O teste de Pearson revelou que os comportamentos em estabilização do bebê estiveram positivamente correlacionados com os comportamentos maternos de tocar ou estimular o bebê com objetos $(\mathrm{r}=0,26 ; p<0,05)$ e negativamente correlacionados com os comportamentos maternos de agir de forma intrusiva $(\mathrm{r}=-0,22 ; p<0,05)$. Assim, quanto mais frequentes foram os comportamentos da mãe de tocar ou estimular o bebê com objetos e menos frequentes foram os comportamentos de agir de forma intrusiva, mais os bebês apresentaram comportamentos em estabilização, que são aqueles mais comumente esperados em crianças com três meses de vida. As demais correlações entre os comportamentos maternos e o desenvolvimento do bebê não foram significativas.

Uma análise de regressão (stepwise) avaliou o poder preditivo da estimulação tátil e com objetos, bem como da intrusividade sobre o total de comportamentos em estabilização apresentados pelo bebê. Os resultados confirmaram o poder preditivo da estimulação tátil e com objetos, que explicou $5,5 \%\left(\mathrm{R}^{2}=0,055\right)$ da variância na frequência de comportamentos em estabilização apresentados pelo bebê $(F(1)=4,65, \mathrm{p}=0,03)$. A cada ocorrência a mais de estimulação tátil e com objetos, o número total de comportamentos em estabilização aumentou em 0,003 unidades. A intrusividade materna foi excluída do modelo $(\mathrm{p}=0,48)$. Esses resultados, ao mesmo tempo em que destacam o poder preditivo da estimulação tátil e com objetos nos comportamentos em estabilização do bebê, não confirmam o possível impacto negativo da intrusividade sobre este aspecto do desenvolvimento do bebê.

\section{Discussão}

As hipóteses deste estudo foram parcialmente confirmadas. A primeira hipótese previa que maiores escores de depressão e de transtornos mentais comuns seriam preditores da menor frequência de comportamentos maternos sensíveis e da maior frequência de comportamentos intrusivos da mãe. Apenas os comportamentos sensíveis de sorrir para o bebê e de tocar ou estimular o bebê estiveram negativamente correlacionados tanto aos sintomas de depressão quanto aos transtornos mentais comuns. Contudo, as análises de regressão confirmaram apenas o poder preditivo dos sintomas de depressão sobre a frequência de sorrisos da mãe.

Esses achados são respaldados por diferentes estudos que examinaram o impacto da depressão pósparto sobre a interação mãe-bebê no primeiro ano de vida. Em virtude das alterações no humor, características da depressão, as mães, além de apresentarem maior dificuldade na regulação das próprias emoções, tendem a apresentar um padrão de sensibilidade, animação e tonicidade prejudicados durante as interações e, em geral, são pouco influenciadas pelos sinais do bebê (Braarud et al., 2017; Brummelte \& Galea, 2016; Field, 2010; Frizzo \& Piccinini, 2005). Assim, mesmo em interações lúdicas com o recém-nascido, é possível que as mães com depressão não respondam de forma mutuamente recíproca, especialmente no que se refere à apresentação de comportamentos que expressam afeto positivo e satisfação, como é o caso do sorriso (Hummel, Kiel, \& Zvirblyte, 2016). Ademais, também é comum que mães deprimidas relatem mais dificuldades e menos satisfação nos cuidados com o filho, o que, por sua vez, pode repercutir em prejuízos para a interação da díade mãe-bebê (Frizzo \& Piccinini, 2005). De qualquer modo, mesmo nos contextos de depressão pós-parto é possível que em alguns momentos a mãe consiga interagir com qualidade com a criança. No entanto, a oscilação entre a adequada disponibilidade emocional e a indisponibilidade materna pode gerar nos bebês um ciclo de expectativa e frustração com relação às interações com a mãe, o que dificulta o desenvolvimento de um padrão de apego seguro (Bowlby, 1969), e consequentemente, o desenvolvimento da criança. 
Apesar de a análise de regressão não ter confirmado o impacto da depressão sobre o comportamento materno de tocar ou estimular o bebê com objetos, a correlação encontrada merece ser explorada e corrobora achados de estudos prévios. Comparadas às mães sem prejuízos emocionais, mães com sintomas de depressão tendem tocar menos o bebê (Field, 2010). Assim, de modo geral, esses dados corroboram outros estudos que enfatizam que os prejuízos da depressão nos comportamentos maternos envolvem a redução na afetividade e no envolvimento da mãe com o bebê (Choi, 2013; Hummel et al., 2016). Uma metanálise de estudos de intervenções sobre a sensibilidade de mães deprimidas durante as interações com o bebê revelou achados interessantes acerca da importância da estimulação tátil. Os resultados mostraram que as intervenções que incluíam técnicas nas quais as mães eram estimuladas a realizar massagens no bebê foram mais eficazes em benefício da sensibilidade materna do que outras intervenções oferecidas, como, por exemplo, a terapia individual para as mães (Kersten-Alvarez, Hosman, Riksen-Walraven, Doesum, \& Hoefnagels, 2011). Esses dados sugerem que os comportamentos maternos de tocar o bebê podem amenizar os efeitos deletérios da depressão pós-parto sobre a interação mãebebê, destacando a importância da estimulação tátil para a relação da díade nos primeiros meses de vida.

De acordo com a segunda hipótese, a maior frequência de comportamentos maternos sensíveis e a menor frequência de comportamentos maternos intrusivos seriam preditores de maiores escores dos indicadores de desenvolvimento da criança. Inicialmente os resultados revelaram uma correlação positiva entre os comportamentos infantis em estabilização e os comportamentos maternos de tocar ou estimular o bebê com objetos e uma correlação negativa entre esse mesmo tipo de comportamento infantil e a resposta materna de agir de forma intrusiva. A análise de regressão confirmou apenas o poder preditivo da estimulação tátil e com objetos sobre os comportamentos em estabilização da criança. Para bebês de três meses de vida, o agrupamento de comportamentos em estabilização é predominantemente composto por comportamentos motores, como por exemplo, levar a mão à boca ou seguir um objeto visualmente até 180 graus. Desse modo, este achado indica a importância da estimulação tátil ou com objetos em favor do desenvolvimento motor do lactente.

Outros estudos com bebês prematuros ou nascidos a termo também indicaram que a estimulação tátil do bebê tende a repercutir na melhor qualidade do desenvolvimento motor (Fucile \& Gisel, 2010; Sayed, Youssef, Hassanein, \& Mobarak, 2015). A influência dos comportamentos maternos sobre o desenvolvimento motor do bebê está intrinsecamente relacionada à qualidade da estimulação, bem como da segurança e precisão nos movimentos que a mãe realiza ao manejar o bebê (Pereira et al., 2016; LevEnacab, Sher-Censor, Einspiele, Daube-Fishman, \& Beni-Shrem, 2015). No presente estudo, a categoria toca ou estimula com objetos envolveu tanto os comportamentos que apenas promoviam o movimento (de mexer nos braços e pernas do bebê, por exemplo) quanto os comportamentos que também favoreciam a apresentação, oferta ou exploração de brinquedos. Nesse sentido, é possível que esses comportamentos maternos tenham beneficiado tanto o desenvolvimento motor da criança, quanto o vínculo entre a díade, que proporciona ao bebê a segurança necessária para explorar o ambiente(Alvarenga etal.,2016; Isabella etal., 1989).Ademais, essas práticas maternas se tornam ainda mais relevantes porque o desenvolvimento motor tem sido particularmente associado ao desenvolvimento cognitivo e, assim, os problemas ou atrasos no desenvolvimento motor podem acarretar atrasos concomitantes no desenvolvimento da atenção e da memória, por exemplo (Pereira et al., 2016). Embora o desenvolvimento das capacidades motoras também esteja associado a outros fatores como, por exemplo, a renda da família (Clearfield et al., 2015), a sensibilidade da mãe pode, até certo ponto, compensar as limitações no acesso a maior variedade de brinquedos ou objetos (Saccani et al., 2013).

Apesar de a intrusividade materna não ter sido confirmada como um preditor do desenvolvimento motor, a correlação inicialmente verificada neste estudo, que indicou prejuízos no desenvolvimento motor em bebês de mães que mais frequentemente agiam de forma intrusiva, apoia os achados de investigações prévias sobre respostas maternas intrusivas e seu impacto sobre o desenvolvimento do bebê. Estudos anteriores enfatizaram os efeitos prejudiciais dos comportamentos maternos intrusivos sobre os diversos domínios do desenvolvimento (Isabella et al., 1989; Field, 2010; Weikum et al., 2013). Em interações com esse tipo de característica os bebês podem apresentar atrasos ou problemas no desenvolvimento especialmente porque as mães tendem a interromper seus comportamentos, limitando assim suas oportunidades para praticar e adquirir novas habilidades (Alvarenga et al., 2016).

Este estudo deve ser apreciado considerando-se algumas limitações. A Escala de Desenvolvimento do Comportamento da Criança, utilizada para avaliar o desenvolvimento infantil, foi desenvolvida a partir da revisão de diversos instrumentos anteriores e foi padronizada para crianças brasileiras (Pinto et al., 1997), o que garantiu a adequação psicométrica do instrumento para essa população. No entanto, este instrumento não foi 
revisado e atualizado nos últimos anos. Considerando as mudanças sociais que envolvem o tratamento e os cuidados com bebês, é possível considerar que algumas mudanças importantes nos valores referenciais do instrumento tenham ocorrido. Uma outra limitação está relacionada ao desenho metodológico dessa investigação. Estudos transversais fornecem informações relevantes sobre as características de um grupo em um momento específico. Porém, especialmente no que se refere à saúde mental materna e ao desenvolvimento infantil, avaliações longitudinais garantiriam dados mais robustos sobre a evolução da depressão e do desenvolvimento, como por exemplo, o desencadeamento de outros problemas emocionais em decorrência da depressão pós-parto e as repercussões, a longo prazo, dos transtornos mentais da mãe sobre o desenvolvimento do bebê.

Por fim, pode-se concluir que os resultados desse estudo ressaltam o impacto da depressão pós-parto sobre uma dimensão afetiva da interação mãe-bebê, o sorriso da mãe, e os efeitos da estimulação tátil e com objetos sobre o desenvolvimento motor. Esses achados mostram que o estado emocional da mãe e seus efeitos comportamentais têm potencial de afetar o desenvolvimento do bebê já nos primeiros meses de vida e, por essa razão, devem ser alvo de avaliação e intervenção em serviços de saúde.

\section{Referências}

Alvarenga, P., Dazzani, M. V. M., Lordelo, E. da R., Alfaya, C. A. dos S., \& Piccinini, C. A. (2013). Predictors of sensitivity in mothers of 8- month-old infants. Paidéia, 23(56), 311-319. https://doi.org/10.1590/198243272356201305

Alvarenga, P., Weber, L. D., \& Bolsoni-Silva, A. (2016). Cuidados parentais e desenvolvimento socioemocional na infância e na adolescência: Uma perspectiva analítico-comportamental. Revista Brasileira de Terapia Comportamental E Cognitiva, 18(1), 4-21. https://doi.org/10.1017/CBO9781107415324.004

American Psychiatric Association (APA) (2014). Manual diagnóstico e estatístico de transtornos mentais (DSM-5) (5 $5^{\mathrm{a}}$ ed.). Porto Alegre: Artmed.

Bayley, N. (2006). Bayley Scales of Infant and Toddler Development: Administration Manual (3a ed.). San Antonio, TX: The Psychological Corporation.

Beck, A. T. \& Steer, R. A. (1993). Beck Depression Inventory. Manual. San Antonio, CA: Psychological Corporation.

Bornstein, M. H., Putnick, D. L., Cote, L. R., Haynes, O. M., \& Suwalsky, J. T. D. (2015). Mother-infant contingent vocalizations in 11 countries. Psychological Science, 26(8), 1272-1284. https://doi.org/10.1177/0956797615586796

Bornstein, M. H., Tamis-LeMonda, C. S., Chun-Shin, H., \& Haynes, O. M. (2008). Maternal responsiveness to young children at three ages: Longitudinal analysis of a multidimensional, modular, and specific parenting construct. Developmental Psychology, 44 (3), 867-874 https://doi.org/10.1037/0012-1649.44.3.867

Bowlby, J. (1969). Attachment and loss: Attachment (Vol. 1). New York: Basic Books.

Braarud, H. C., Skotheim, S., Høie, K., Markhus, M. W., Kjellevold, M., Graff, I. E., ..., \& Stormark, K. M. (2017). Affective facial expression in sub-clinically depressed and non-depressed mothers during contingent and noncontingent face-to-face interactions with their infants. Infant Behavior and Development, 48, 98-104. https://doi. org/10.1016/j.infbeh.2017.05.004

Brummelte, S. \& Galea, L. A. M. (2016). Postpartum depression: Etiology, treatment and consequences for maternal care. Hormones and Behavior, 77, 153-166. https://doi.org/10.1016/j.yhbeh.2015.08.008

Choi, E. (2013). An exploratory study of maternal depressive symptoms and infant's social skills in Korea: The mediating role of mothers' social interactions with their infants. Infant Mental Health Journal, 34(5), 458-469. https://doi.org/10.1002/imhj.21397

Clearfield, M. W., Stanger, S. B., \& Jenne, H. K. (2015). Socioeconomic status (SES) affects means-end behavior across the first year. Journal of Applied Developmental Psychology, 38, 22-28. https://doi.org/10.1016/j.appdev.2015.02.001

Cunha, J. A. (2001). Escalas Beck. São Paulo: Casa do Psicólogo.

Edwards, R. C. \& Hans, S. L. (2016). Prenatal depressive symptoms and toddler behavior problems: The role of maternal sensitivity and child sex. Child Psychiatry \& Human Development, 47(5), 696-707. https://doi.org/10.1007/ s10578-015-0603-6

Field, T. (2010). Postpartum depression effects on early interaction, parenting, and safety practices: A review. Infant Behavior and Development, 33, 1-6. https://doi.org/10.1016/j.infbeh.2009.10.005

Fucile, S., \& Gisel, E. G. (2010). Sensorimotor interventions improve growth and motor function in preterm infants. Neonatal Network, 29(6), 359-366. https://doi.org/10.1891/0730-0832.29.6.359

Gonçalves, D. M., Stein, A. T., \& Kapczinski, F. (2008). Avaliação de desempenho do Self-Reporting Questionnaire como instrumento de rastreamento psiquiátrico: Um estudo comparativo com o Structured Clinical Interview for DSM-IV-TR. Cadernos de Saúde Pública, 24, 380-390. https://doi.org/10.1590/S0102-311X2008000200017

Hummel, A. C., Kiel, E. J., \& Zvirblyte, S. (2016). Bidirectional effects of positive affect, warmth, and interactions between mothers with and without symptoms of depression and their toddlers. Journal of Child and Family Studies, 5(3), 781-789. https://doi.org/10.1007/s10826-015-0272-x 
Isabella, R. A., Belsky, J., \& Von Eye, A. (1989). Origins of infant-mother attachment: An examination of interactional synchrony during the infant's first year. Developmental Psychology, 25(1), 12-21. https://doi.org/10.1037/00121649.25.1.12

Frizzo, G. B. \& Piccinini, C. A. (2005). Interação mãe-bebê em contexto de depressão materna: Aspectos teóricos e empíricos. Psicologia em Estudo, 10(1), 47-55. https://doi.org/10.1590/S0103-166X2005000200004

Karam, F., Sheehy, O., Huneau, M., Chambers, C., Fraser, W. D., Johnson, D., ..., \& Lavigne, S. V. (2016). Impact of maternal prenatal and parental postnatal stress on 1-year-old child development: Results from the OTIS antidepressants in pregnancy study. Archives of Women's Mental Health, 835-843. https://doi.org/10.1007/s00737016-0624-6

Kersten-Alvarez, L. E., Hosman, C. M. H., Riksen-Walraven, J. M., Doesum, K. T. M. Van, \& Hoefnagels, C. (2011). Which preventive interventions effectively enhance depressed mothers' sensitivity? A meta-analysis. Infant Mental Health Journalental Health Journal, 32(3), 362-376. https://doi.org/10.1002/imhj.20301

Kingston, D., Tough, S., \& Whitfield, H. (2012). Prenatal and postpartum maternal psychological distress and infant development: A systematic review. Child Psychiatry and Human Development, 43(5), 683-714. https://doi. org/10.1007/s10578-012-0291-4

Koutra, K., Chatzi, L., Bagkeris, M., Vassilaki, M., Bitsios, P., \& Kogevinas, M. (2013). Antenatal and postnatal maternal mental health as determinants of infant neurodevelopment at 18 months of age in a mother-child cohort (Rhea Study) in Crete, Greece. Social Psychiatry and Psychiatric Epidemiology, 48(8), 1335-1345. https://doi. org/10.1007/s00127-012-0636-0

Lev-Enacab, O., Sher-Censor, E., Einspieler, C., Daube-Fishman, G., \& Beni-Shrem, S. (2015). The quality of spontaneous movements of preterm infants: Associations with the quality of mother-infant interaction. Infancy, 20(6), 634-660. https://doi.org/10.1111/infa.12096

Lobato, G., Moraes C. L., \& Reichenheim, M. E. (2011). Magnitude da depressão pós-parto no Brasil: Uma revisão sistemática. Revista Brasileira de Saúde Materno-Infantil, 11(4), 369-379. https://doi.org/10.1590/S151938292011000400003

Mari, J. \& Williams, P. (1986). A validity study of a psychiatric screening questionnaire (SRQ-20) in primary care in the city of São Paulo. British Journal of Psychiatry, 148, 23-26. https://doi.org/10.1192/bjp.148.1.23

Mermelshtine, R. \& Barnes, J. (2016). Maternal responsive-didactic caregiving in play interactions with 10-month-olds and cognitive development at 18 months. Infant and Child Development, 25(3), 296-316. https://doi.org/10.1002/icd

Parfitt, Y., Pike, A., \& Ayers, S. (2013). The impact of parents' mental health on parent-baby interaction: A prospective study. Infant Behavior and Development, 36(4), 599-608. https://doi.org/10.1016/j.infbeh.2013.06.003

Pereira, K. R. G., Valentini, N. C., \& Saccani, R. (2016). Brazilian infant motor and cognitive development: Longitudinal influence of risk factors. Pediatrics International, 58(12), 1297-1306. https://doi.org/10.1111/ped.13021

Piccinini, C. A., Alvarenga, P., \& Frizzo, G. B. (2007). A responsividade como foco da análise da interação mãebebê e pai-bebê. In C. A. Piccinini \& M. L. Seidl de Moura (Orgs.). Observando as interações pais-bebê-criança (pp. 131-153). Itatiba: Casa do Psicólogo.

Pinto, E. B., Vilanova, L. C. P., \& Vieira, R. M. (1997). O desenvolvimento do comportamento da criança no primeiro ano de vida: padronização de uma escala para a avaliação e o acompanhamento. São Paulo: Casa do Psicólogo: FAPESP.

Robson, C. (1993). Real World Research, Oxford: Cambridge: Blackwell.

Saccani, R., Valentini, N. C., Pereira, K. R. G., Müller, A. B., \& Gabbard, C. (2013). Associations of biological factors and affordances in the home with infant motor development. Pediatrics International, 55(2), 197-203. https://doi. org/10.1111/ped.12042

Santos, I. S., Matijasevich, A., Tavares, B. F., Lima, A. C. C., Riegel, R. E., \& Lopes, B. C. (2007). Comparing validity of Edinburgh scale and SRQ20 in screening for post-partum depression. Clinical Practice and Epidemiology in Mental Health, 3(18), 1-5. https://doi.org/10.1186/1745-0179-3-18

Sayed, A. M. S., Youssef, M. M. E., Hassanein, F. E., \& Mobarak, A. A. (2015). Impact of tactile stimulation on neurobehavioral development of premature infants in Assiut City. Journal of Education and Practice, 8(6), 93-102.

Weikum, W. M., Mayes, L. C., Grunau, R. E., Brain, U., \& Oberlander, T. F. (2013). The impact of prenatal serotonin reuptake inhibitor (SRI) antidepressant exposure and maternal mood on mother-infant interactions at 3 months of age. Infant Behavior and Development, 36(4), 485-493. https://doi.org/10.1016/j.infbeh.2013.04.001

\footnotetext{
Dados dos autores:

Patrícia Alvarenga - Doutora, Universidade Federal do Bahia

Catiele Paixão, Doutoranda - Universidade Federal do Bahia.

Zelma Freitas Soares - Mestranda, Universidade Federal do Bahia.

Endereço para correspondência:

Patrícia Alvarenga

Instituto de Psicologia, Universidade Federal da Bahia

Rua Aristides Novis, 197 - Estrada de São Lázaro

40210-730 Salvador, BA, Brasil

<palvarenga66@gmail.com>
}

Antonio Carlos Santos da Silva - Mestrando, Universidade Federal do Bahia.

Recebido em: 03.09.2017

Aceito em: 15.01.2018 